\title{
BMJ Open Synergistic effect of interaction between perceived health and social activity on depressive symptoms in the middle- aged and elderly: a population-based longitudinal study
}

\author{
Sung-Youn Chun, ${ }^{1,2}$ Kyu-Tae Han, ${ }^{1,2}$ Seo Yoon Lee, ${ }^{2,3}$ Chan Ok Kim, ${ }^{1,2}$ \\ Eun-Cheol Park ${ }^{2,4}$
}

To cite: Chun S-Y, Han K-T, Lee SY, et al. Synergistic effect of interaction between perceived health and social activity on depressive symptoms in the middle-aged and elderly: a populationbased longitudinal study. BMJ Open 2015;5:e007154. doi:10.1136/bmjopen-2014007154

- Prepublication history for this paper is available online. To view these files please visit the journal online (http://dx.doi.org/10.1136/ bmjopen-2014-007154).

Received 10 November 2014 Revised 29 January 2015 Accepted 16 February 2015

CrossMark

For numbered affiliations see end of article.

Correspondence to Dr Eun-Cheol Park; ecpark@yuhs.ac

\section{ABSTRACT}

Objective: To examine the synergistic effect of interaction between perceived health and social activity on depressive symptoms.

Methods: We investigated whether the interaction between perceived health and social activity has a synergistic effect on depressive symptoms in the middle-aged and elderly using data from 6590 respondents aged 45 and older in the Korean Longitudinal Study on Aging (KLoSA), 2006-2012.

A generalised linear mixed-effects model was used to investigate the association in a longitudinal data form. Depressive symptoms were measured using the Center for Epidemiological Studies Depression 10 Scale (CES-D10). Perceived health and level of social activity were categorical variables with three values. Participation in six social activities was assessed.

Results: Interactions between perceived health status and social activity were statistically significant for almost all social activity/perceived health combinations. Addition of the interaction term significantly decreased CES-D10 scores, confirming the synergistic effect of the interaction between perceived health status and social activity ('normal $\times$ moderate', $\beta=-0.1826$; 'poor $\times$ moderate', $\beta=-0.5739$; 'poor $\times$ active', $\beta=-0.8935$ ). In addition, we performed stratified analyses by region: urban or rural. In urban respondents, the additional effect of the interaction term decreased CES-D10 scores and all social activity/perceived health combinations were statistically significant ('normal×moderate', $\beta=-0.2578$; 'normal xactive', $\beta=-0.3945$; 'poor $\times$ moderate', $\beta=-0.5739$; 'poor $\times$ active', $\beta=-0.8935)$. In rural respondents, only one social activity/perceived health combination was statistically significant, and the additional effect of the interaction term showed no consistent trend on CES-D10 scores.

Conclusions: The interaction between perceived health and social activity has a synergistic effect on depressive symptoms; the additional effect of the interaction term significantly decreased CES-D10 scores in our models.

\section{Strengths and limitations of this study}

- To the best of our knowledge, it is the first to demonstrate the effect of an interaction between perceived health and social activity on depressive symptoms.

- It analysed data from a nationally representative sample.

- It had a large sample size.

- Social activity is just one way to measure social capital. Social networks, social support and social relationships were not considered in this study.

- The frequency of social activity was not considered.

\section{INTRODUCTION}

We are living in an aging society. In Organization for Economic Cooperation and Development (OECD) countries, the proportion of the population aged 65 years and older has increased from less than $9 \%$ in 1960 to $15 \%$ in 2010 and is expected to nearly double in the next four decades, reaching $27 \%$ in 2050 . In Korea, the elderly comprised $11 \%$ of the population in 2010 and are expected to exceed $37 \%$ in $2050 .{ }^{1}$ In an aging society, the health and welfare of the elderly become major concerns.

Depression in later life has a substantial impact on affected individuals and their families. ${ }^{2}$ Depression is treatable, but individuals often have a hard time recognising it and obtaining proper treatment. ${ }^{3}$ Many studies have attempted to identify risk factors for depression. One prognostic indicator is self-rated perceived health. Those who report poor perceived health have greater odds of developing depression than those who report good perceived health. ${ }^{4}$ In Korea, the percentage of adults who report good perceived health is $36.8 \%$, 
second to last among OECD countries. ${ }^{1}$ The need for intervention is clear.

Social activity is inversely associated with depression. ${ }^{5}$ For example, one study reported that a higher level of social activity is inversely associated with depressive symptoms in later life and positively associated with symptom improvement. ${ }^{6}$ In addition, social isolation and lack of a social network are important risk factors for depression. ${ }^{78}$ Thus, previous studies have shown that perceived health is positively associated with depression, and social activity is inversely associated with depression. However, whether social activity and perceived health status work together to moderate depressive symptoms is poorly understood.

We hypothesised that, when a statistical interaction between perceived health and social activity is present, a higher level of social activity will be more strongly associated with a decrease in depressive symptoms in those who reported poor perceived health. Thus, the purpose of this study was to examine whether social activity level has a synergistic effect on the relationship between perceived health and depression. A previous study found that factors associated with depression differed between urban and rural areas. ${ }^{9}$ Therefore, we also performed an analysis stratified by location.

\section{METHODS}

This study used data from the Korean Longitudinal Study on Aging (KLoSA). KLoSA is a national, representative, longitudinal study that follows-up participants every 2 years. The Korea Labor Institute conducts KLoSA, which is funded by the Korean Ministry of Labor, to investigate the aging process in Koreans and to use its findings in academic research and policy-making. Household members aged 45 and older were recruited using multistage probability sampling methods. As the KLoSA data are secondary data that do not contain private information and are available in the public domain, our study did not need to address ethical concerns. We drew on four waves of data collection, conducted between 2006 and 2012. In the 2006 baseline survey, trained interviewers interviewed 10254 individuals from 6171 households (1.7 individuals per household) using a computer-assisted personal interviewing method. The second survey, in 2008, followed-up 8688 subjects, representing $86.6 \%$ of the original panel, with the exception of 187 decedents. The third survey, in 2010, followed-up 7920 subjects, representing $81.7 \%$ of the second wave, with the exception of 309 decedents. Finally, the fourth survey, in 2012, followed-up 7486 subjects, representing $80.1 \%$ of the third survey, with the exception of 327 decedents. We excluded newly added participants and decedents, as well as 896 participants with missing sociodemographic data. We used the first to fourth wave in KLoSA, which is four waves in 2006-2012, so there are four repeated measures for each individual. In total, we analysed data from 6590 participants aged 45 and older.

\section{Outcome measure: depressive symptoms}

Depressive symptoms were assessed using the Korean version of the Center for Epidemiological Studies Depression 10 Scale (CES-D10). This self-report scale was designed to measure depressive symptoms in the general population. The items assess the presence of the following features over the previous month: feelings of depression, pessimism, irritability, tearfulness, fatigue, sleep problems, loss of interest, loss of appetite, reduced concentration, and loss of enjoyment. KLoSA's CES-D10 used a yes or no response format. Ten items were added together, resulting in a composite score ranging from 0 to 10 which was used as a continuous variable. On this scale, zero represents perfect mental health and 10 represents severe depression. The Boston version of the CES-D10 reported $\alpha$ coefficients of 0.80 for older adults ${ }^{10}$ and 0.92 for middle-aged individuals. ${ }^{11}$

\section{Independent variables}

In this study, the primary independent variable was the interaction term between social activity and perceived health status. Interaction terms present synergistic effects of perceived health and social activity on depressive symptoms.

$$
\begin{aligned}
\mathrm{CES}-D 10= & (\text { Perceived health }) \times \beta 1+(\text { Social activity }) \times \beta 2 \\
& +(\text { Perceived health } \times \text { Socialactivity }) \times \beta 3 \\
& + \text { covariates }
\end{aligned}
$$

In KLoSA, the social activity variable comprised six activities: 'religious organisations', 'friendship organisations', 'leisure/culture/sports clubs', 'school/family reunions', 'voluntary work' and 'political organisations'. Respondents who did not participate in any of these social activities were grouped in the 'none' category, respondents who participated in one or two social activities were grouped in the 'moderate' category, and respondents who participated in three or more activities were grouped in the 'active' category. Perceived health status was measured using a five-point scale: 'very good', 'good', 'normal', 'poor' and 'very poor'. Before analysis, these values were converted into three categories: 'good', 'normal' and 'poor'.

Covariates considered in this study were sex, age, household income, marital status, educational level, region (urban, rural), employment status, smoking status and alcohol use.

We also stratified analyses by region (urban, rural).

\section{Statistical analysis}

Analysis of variance was used to analyse statistical differences with regard to CES-D10 scores. A generalised linear mixed-effects model was used to evaluate factors influencing depressive symptoms among middle-aged and elderly participants from 2006 to 2012 and, in particular, to analyse the associations between social activity, perceived health and depressive symptoms while 
Table 1 General sample characteristics at baseline (2006)

\begin{tabular}{|c|c|c|}
\hline Characteristic & $\begin{array}{l}\text { Number } \\
(\mathrm{N}=6590)\end{array}$ & Percentage \\
\hline \multicolumn{3}{|l|}{ Perceived health status } \\
\hline Good & 2597 & 39.4 \\
\hline Normal & 2128 & 32.3 \\
\hline Poor & 1865 & 28.3 \\
\hline \multicolumn{3}{|l|}{ Number of social activities } \\
\hline None & 1667 & 25.3 \\
\hline Moderate & 4409 & 66.9 \\
\hline Active & 514 & 7.8 \\
\hline \multicolumn{3}{|l|}{ Sex } \\
\hline Male & 2859 & 43.4 \\
\hline Female & 3731 & 56.6 \\
\hline \multicolumn{3}{|l|}{ Age (years) } \\
\hline $45-54$ & 2188 & 33.2 \\
\hline $55-64$ & 2016 & 30.6 \\
\hline $65-74$ & 1736 & 26.3 \\
\hline $75-84$ & 596 & 9.0 \\
\hline$>85$ & 54 & 0.8 \\
\hline \multicolumn{3}{|l|}{ Education } \\
\hline College graduate & 630 & 9.6 \\
\hline High school graduate & 1799 & 27.3 \\
\hline$<$ High school & 4161 & 63.1 \\
\hline \multicolumn{3}{|l|}{ Income } \\
\hline Q4 (high) & 1638 & 24.9 \\
\hline Q3 & 1537 & 23.3 \\
\hline Q2 & 1729 & 26.2 \\
\hline Q1 (low) & 1686 & 25.6 \\
\hline \multicolumn{3}{|l|}{ Marital status } \\
\hline Married & 5419 & 82.2 \\
\hline Divorced, widowed, unmarried & 1171 & 17.8 \\
\hline \multicolumn{3}{|l|}{ Type of household } \\
\hline With family & 6070 & 92.1 \\
\hline Alone & 520 & 7.9 \\
\hline \multicolumn{3}{|l|}{ Chronic disease } \\
\hline No & 3592 & 54.5 \\
\hline Yes & 2998 & 45.5 \\
\hline \multicolumn{3}{|l|}{ Job status } \\
\hline Yes & 2747 & 41.7 \\
\hline No & 3843 & 58.3 \\
\hline \multicolumn{3}{|l|}{ Smoking status } \\
\hline Current smoker & 1254 & 19.0 \\
\hline Ex-smoker & 634 & 9.6 \\
\hline Non-smoker & 4702 & 71.4 \\
\hline \multicolumn{3}{|l|}{ Alcohol use } \\
\hline Current & 2587 & 39.3 \\
\hline Past & 395 & 6.0 \\
\hline Never & 3608 & 54.8 \\
\hline \multicolumn{3}{|l|}{ Region } \\
\hline Urban & 4846 & 73.5 \\
\hline Rural & 1744 & 26.5 \\
\hline
\end{tabular}

accounting for the longitudinal nature of the data, which required the incorporation of a correlated structure among observations. We used an 'unstructured' covariance structure, so that we could consider all covariance within subjects. All statistical analyses were carried out using SAS V.9.2.

\section{RESULTS}

The characteristics of the study participants at baseline (2006) are shown in table 1 . Of the 6590 participants, 1865 people reported poor, 2128 people reported normal, and 2597 people reported good perceived health. In addition, 1667 people did not participate in any social activity at all, 4409 people were moderately socially active, and 514 people were active.

Table 2 presents mean CES-D10 scores stratified by social activity, perceived health and other sociodemographic characteristics for each survey year. In terms of health, people with poor perceived health showed the highest CES-D10 scores (4.52 in 2006, 5.38 in 2008, 5.23 in 2010, and 5.21 in 2012), and people with good perceived health showed the lowest scores (1.80 in 2006, 2.36 in 2008, 2.57 in 2010, and 2.61 in 2012). In terms of social activity, active people had the lowest scores (1.67 in 2006, 1.87 in 2008, 2.45 in 2010, and 2.46 in 2012), and individuals who did not participate in any social activities had the highest (3.69 in 2006, 4.67 in 2008, 4.68 in 2010, and 4.71 in 2012). In addition, men had lower scores than women, and scores tended to increase with age. Finally, CES-D10 scores decreased as education level and household income increased.

Table 3 presents the generalised linear mixed-effects model results of waves one to four of KLoSA, which assessed the associations between social activity, perceived health and depression among the middle-aged and elderly. When all covariates were included in the model, CES-D10 scores differed significantly between individuals at each level of perceived health. Individuals with poor perceived health were more likely to have higher CES-D10 scores $(\beta=2.1397)$ than individuals with good perceived health. In addition, individuals with normal perceived health were more likely to have higher scores than individuals with good perceived health $(\beta=0.6258)$. Respondents who were socially 'active' were more likely to have lower scores than those with no social activity participation $(\beta=-0.3756)$. 'Moderate' respondents were also more likely to have lower scores than those with no social activity participation $(\beta=-0.0711, \mathrm{p}>0.05)$, but this difference was not statistically significant.

The interaction between perceived health status and social activity was statistically significant in all health/ social activity combinations except 'normal perceived health $\times$ active social activity' $(\beta=-0.281, \quad \mathrm{p}>0.05)$. Furthermore, addition of the interaction term significantly decreased CES-D10 scores, confirming the synergistic effect of the interaction between perceived health status and social activity. In individuals with poor perceived health, addition of the interaction term with social activity decreased CES-D10 score more ('normal $\times$ less active', $\beta=-0.1826$; 'poor $\times$ moderate', $\beta=-0.5739$; and 'poor $\times$ active', $\beta=-0.8935$ ).

Table 4 presents an analysis of waves one to four of KLoSA stratified by region: urban or rural. In urban respondents, addition of the interaction term significantly decreased CES-D10 scores for all health/social 
Table 2 Mean CES-D10 depression scores of participants

\begin{tabular}{|c|c|c|c|c|c|c|c|c|c|c|c|c|}
\hline \multirow[b]{2}{*}{ Characteristic } & \multicolumn{3}{|l|}{2006} & \multicolumn{3}{|l|}{2008} & \multicolumn{3}{|l|}{2010} & \multicolumn{3}{|l|}{2012} \\
\hline & Mean & SD & p Value & Mean & SD & p Value & Mean & SD & p Value & Mean & SD & p Value \\
\hline \multicolumn{13}{|l|}{ Perceived health status } \\
\hline Good & 1.80 & 1.76 & \multirow[t]{3}{*}{$<0.0001$} & 2.36 & 2.36 & \multirow[t]{3}{*}{$<0.0001$} & 2.57 & 2.50 & \multirow[t]{3}{*}{$<0.0001$} & 2.61 & 2.45 & \multirow[t]{3}{*}{$<0.0001$} \\
\hline Normal & 2.65 & 2.26 & & 3.53 & 2.74 & & 3.40 & 2.82 & & 3.16 & 2.72 & \\
\hline Poor & 4.52 & 2.93 & & 5.38 & 2.88 & & 5.23 & 3.01 & & 5.21 & 3.06 & \\
\hline \multicolumn{13}{|l|}{ Social activity } \\
\hline None & 3.69 & 2.94 & \multirow[t]{3}{*}{$<0.0001$} & 4.67 & 3.02 & \multirow[t]{3}{*}{$<0.0001$} & 4.68 & 3.05 & \multirow[t]{3}{*}{$<0.0001$} & 4.71 & 3.17 & \multirow[t]{3}{*}{$<0.0001$} \\
\hline Moderate & 2.66 & 2.38 & & 3.43 & 2.81 & & 3.34 & 2.87 & & 3.27 & 2.79 & \\
\hline Active & 1.67 & 1.78 & & 1.87 & 2.23 & & 2.45 & 2.65 & & 2.46 & 2.58 & \\
\hline \multicolumn{13}{|l|}{ Sex } \\
\hline Male & 2.44 & 2.31 & \multirow[t]{2}{*}{0.0114} & 3.10 & 2.77 & 0.0091 & 3.27 & 2.88 & 0.5417 & 3.30 & 2.88 & 0.9096 \\
\hline Female & 3.16 & 2.70 & & 4.00 & 2.94 & & 3.94 & 3.01 & & 3.83 & 2.99 & \\
\hline Age (years) & & & & & & & & & & & & \\
\hline $45-54$ & 2.30 & 2.22 & $<0.0001$ & 2.80 & 2.57 & 0.0216 & 2.72 & 2.65 & 0.2602 & 2.68 & 2.59 & $<0.0001$ \\
\hline $55-64$ & 2.58 & 2.45 & & 3.22 & 2.78 & & 3.11 & 2.75 & & 2.92 & 2.70 & \\
\hline $65-74$ & 3.46 & 2.75 & & 4.12 & 2.96 & & 3.97 & 3.01 & & 3.69 & 2.95 & \\
\hline $75-84$ & 3.86 & 2.80 & & 4.86 & 2.99 & & 4.66 & 3.06 & & 4.78 & 2.99 & \\
\hline$>85$ & 3.80 & 2.82 & & 5.05 & 2.92 & & 4.65 & 3.15 & & 5.14 & 3.09 & \\
\hline Education & & & & & & & & & & & & \\
\hline College graduate & 1.88 & 1.88 & 0.0163 & 2.46 & 2.39 & 0.0114 & 2.42 & 2.48 & $<0.0001$ & 2.79 & 2.65 & 0.6606 \\
\hline High school graduate & 2.12 & 2.07 & & 2.82 & 2.63 & & 2.89 & 2.70 & & 2.98 & 2.71 & \\
\hline$<$ High school & 3.31 & 2.72 & & 4.13 & 2.96 & & 4.16 & 3.03 & & 4.00 & 3.02 & \\
\hline Income & & & & & & & & & & & & \\
\hline Q4 (high) & 2.02 & 2.01 & $<0.0001$ & 2.78 & 2.57 & $<0.0001$ & 2.77 & 2.68 & $<0.0001$ & 2.87 & 2.69 & 0.0374 \\
\hline Q3 & 2.56 & 2.34 & & 3.09 & 2.74 & & 3.20 & 2.82 & & 3.12 & 2.80 & \\
\hline Q2 & 3.19 & 2.72 & & 3.68 & 2.85 & & 3.70 & 2.96 & & 3.79 & 2.94 & \\
\hline Q1 (low) & 3.55 & 2.78 & & 4.82 & 3.00 & & 4.84 & 2.99 & & 4.56 & 3.06 & \\
\hline Marital status & & & & & & & & & & & & \\
\hline Married & 2.56 & 2.38 & $<0.0001$ & 3.32 & 2.81 & $<0.0001$ & 3.36 & 2.89 & 0.0011 & 3.31 & 2.84 & 0.0008 \\
\hline $\begin{array}{l}\text { Divorced, widowed, } \\
\text { unmarried }\end{array}$ & 4.15 & 2.94 & & 4.80 & 2.99 & & 4.70 & 3.03 & & 4.63 & 3.09 & \\
\hline Type of household & & & & & & & & & & & & \\
\hline With family & 2.72 & 2.48 & 0.7201 & 3.48 & 2.86 & 0.4607 & 3.50 & 2.94 & 0.0753 & 3.46 & 2.92 & 0.0872 \\
\hline Alone & 4.32 & 2.95 & & 4.87 & 2.98 & & 4.97 & 2.93 & & 4.74 & 3.00 & \\
\hline Chronic disease & & & & & & & & & & & & \\
\hline No & 2.35 & 2.24 & 0.1213 & 2.94 & 2.68 & 0.0047 & 2.99 & 2.74 & 0.0758 & 2.90 & 2.65 & 0.0201 \\
\hline Yes & 3.44 & 2.78 & & 4.22 & 2.97 & & 4.13 & 3.05 & & 4.04 & 3.04 & \\
\hline Employment status & & & & & & & & & & & & \\
\hline Yes & 2.28 & 2.23 & $<0.0001$ & 2.91 & 2.62 & $<0.0001$ & 2.99 & 2.74 & $<0.0001$ & 2.75 & 2.59 & $<0.0001$ \\
\hline No & 3.25 & 2.70 & & 4.18 & 3.00 & & 4.16 & 3.04 & & 4.16 & 3.04 & \\
\hline Smoking status & & & & & & & & & & & & \\
\hline Current smoker & 2.75 & 2.60 & 0.0035 & 3.35 & 2.88 & 0.0236 & 3.44 & 2.90 & 0.1735 & 3.32 & 2.86 & 0.3200 \\
\hline Ex-smoker & 2.63 & 2.36 & & 3.28 & 2.75 & & 3.43 & 2.94 & & 3.51 & 2.98 & \\
\hline Non-smoker & 2.90 & 2.57 & & 3.73 & 2.93 & & 3.74 & 2.99 & & 3.69 & 2.96 & \\
\hline Alcohol use & & & & & & & & & & & & \\
\hline Current & 2.53 & 2.39 & 0.0643 & 3.08 & 2.73 & 0.3448 & 3.14 & 2.76 & 0.0317 & 3.04 & 2.72 & 0.0013 \\
\hline Past & 3.49 & 2.85 & & 3.99 & 2.97 & & 4.10 & 3.03 & & 4.22 & 3.09 & \\
\hline Never & 3.00 & 2.61 & & 3.92 & 2.96 & & 3.14 & 2.76 & & 3.81 & 3.00 & \\
\hline Region & & & & & & & & & & & & \\
\hline Urban & 2.72 & 2.53 & 0.0117 & 3.46 & 2.90 & 0.0002 & 3.54 & 2.96 & 0.0641 & 3.53 & 2.99 & 0.2350 \\
\hline Rural & 3.21 & 2.61 & & 4.03 & 2.86 & & 3.96 & 2.99 & & 3.80 & 2.84 & \\
\hline
\end{tabular}

$\mathrm{p}$ Values for results by analysis of variance.

CES-D10, Center for Epidemiological Studies Depression 10 Scale.

activity combinations ('normal $\times$ moderate', $\beta=-0.2578$; 'normal $\times$ active', $\beta=-0.3945$; 'poor $\times$ moderate', $\beta=-0.5739$; and 'poor $\times$ active', $\beta=-0.8935)$. In rural respondents, addition of the interaction term significantly decreased
CES-D10 scores for only one health/social activity combination ('poor $\times$ moderate', $\beta=-0.4379$ ).

Table 5 presents a combined analysis model of association between perceived health, social activity and 
Table 3 Associations between perceived health, social activity, sociodemographic characteristics and CES-D10 depression score

\begin{tabular}{|c|c|c|c|}
\hline & B & SE & p Value \\
\hline \multicolumn{4}{|l|}{ Perceived health status } \\
\hline Good & Ref & - & - \\
\hline Normal & 0.626 & 0.083 & $<0.0001$ \\
\hline Poor & 2.140 & 0.084 & $<0.0001$ \\
\hline \multicolumn{4}{|l|}{ Social activity } \\
\hline None & Ref & - & - \\
\hline Moderate & -0.071 & 0.073 & 0.3298 \\
\hline Active & -0.377 & 0.115 & 0.0011 \\
\hline \multicolumn{4}{|c|}{ Perceived health status×social activity } \\
\hline Good×none & Ref & - & - \\
\hline Normal×moderate & -0.183 & 0.091 & 0.0447 \\
\hline Normalxactive & -0.281 & 0.160 & 0.0797 \\
\hline Poor $\times$ moderate & -0.574 & 0.092 & $<0.0001$ \\
\hline Poorxactive & -0.895 & 0.228 & $<0.0001$ \\
\hline \multicolumn{4}{|l|}{ Sex } \\
\hline Male & Ref & - & - \\
\hline Female & 0.150 & 0.065 & 0.0220 \\
\hline \multicolumn{4}{|l|}{ Age (years) } \\
\hline $45-54$ & Ref & - & - \\
\hline $55-64$ & -0.098 & 0.049 & 0.0482 \\
\hline $65-74$ & 0.071 & 0.061 & 0.2456 \\
\hline $75-84$ & 0.288 & 0.077 & 0.0002 \\
\hline$>85$ & 0.275 & 0.129 & 0.0321 \\
\hline \multicolumn{4}{|l|}{ Education } \\
\hline College graduate & Ref & - & - \\
\hline High school graduate & 0.121 & 0.082 & 0.1414 \\
\hline$<$ High school & 0.374 & 0.083 & $<0.0001$ \\
\hline \multicolumn{4}{|l|}{ Income } \\
\hline Q4 (high) & Ref & - & - \\
\hline Q3 & 0.014 & 0.048 & 0.764 \\
\hline Q2 & 0.193 & 0.050 & 0.0001 \\
\hline Q1 (low) & 0.295 & 0.056 & $<0.0001$ \\
\hline \multicolumn{4}{|l|}{ Marital status } \\
\hline Married & Ref & - & - \\
\hline Divorced, widowed, unmarried & 0.558 & 0.067 & $<0.0001$ \\
\hline \multicolumn{4}{|l|}{ Type of household } \\
\hline With family & Ref & - & - \\
\hline Alone & 0.168 & 0.083 & 0.0425 \\
\hline \multicolumn{4}{|l|}{ Chronic disease } \\
\hline No & Ref & - & - \\
\hline Yes & 0.182 & 0.042 & $<0.0001$ \\
\hline \multicolumn{4}{|l|}{ Employment status } \\
\hline Yes & -0.345 & 0.041 & $<0.0001$ \\
\hline No & Ref & - & - \\
\hline \multicolumn{4}{|l|}{ Smoking status } \\
\hline Current smoker & Ref & - & - \\
\hline Ex-smoker & -0.149 & 0.067 & 0.0272 \\
\hline Non-smoker & -0.170 & 0.066 & 0.0104 \\
\hline \multicolumn{4}{|l|}{ Alcohol use } \\
\hline Current & -0.067 & 0.053 & 0.2041 \\
\hline Past & 0.251 & 0.071 & 0.0004 \\
\hline Never & Ref & - & - \\
\hline \multicolumn{4}{|l|}{ Region } \\
\hline Urban & -0.188 & 0.050 & 0.0002 \\
\hline Rural & Ref & - & - \\
\hline
\end{tabular}

CES-D10. If participants who have good perceived health are socially 'active', they have a 0.377 lower mean CES-D10 score than participants who have good perceived health and do not participate in social activity. Furthermore, if participants who have poor perceived health are socially 'active', they have a 1.272 lower mean CES-D10 score than participants who have poor perceived health and do not participate in social activity.

Figure 1 presents a graph for CES-D10 score of waves one to four of KLoSA by 'perceived health $\times$ social activity' combination. Each bar depicts the adjusted least squares mean for each 'perceived health $\times$ social activity' combination. The adjusted least squares mean of CES-D10 increases when perceived health is poorer, and decreases when the level of social activity is higher.

\section{DISCUSSION}

An association between poor perceived health and depressive symptoms has been reported in many studies, as has an inverse association between social activity and depression. ${ }^{12-15}$ The purpose of this study was to test the hypothesis that social activity can affect the relationship between perceived health and depression. As in previous studies, we found that poor perceived health was significantly associated with a higher CES-D10 score. Several studies have found that poorer perceived health is associated with higher levels of depression symptoms. ${ }^{4}{ }^{16}$ We also found that high levels of social activity were associated with significantly lower CES-D10 scores. Many studies have shown that higher levels of social engagement or social activity are associated with fewer depressive symptoms. ${ }^{6}{ }^{17}$ Other studies have similarly found that higher levels of social integration are associated with a lower risk of depression. ${ }^{18-21}$

Our major finding was that the interaction between perceived health and social activity significantly decreased CES-D10 scores for all perceived health/social activity combinations except one. In participants who reported poor perceived health, the interaction term with social activity was more strongly associated with CES-D10 score. Furthermore, the additional effect of the interaction term decreased CES-D10 scores more when perceived health was poor and social activity levels were high. Thus, we found that social activity has a synergistic effect on the relationship between perceived health and depressive symptoms. In figure 1, the synergistic effect of the interaction term is evident. Each bar in the figure depicts the adjusted least squares mean for each 'perceived health $\times$ social activity' combination. The graph shows that CES-D10 scores decrease as the level of social activity increases for each perceived health category, after adjustment for covariates. The most precipitous decrease associated with social activity was observed in participants with poor perceived health. In table 5 , the total effect of interaction confirms a synergistic effect. Among those who have poor perceived health, people who are socially active have a lower CES-D10 
Table 4 Associations between perceived health, social activity, sociodemographic characteristics and CES-D10 depression score, stratified by region (urban, rural)

\begin{tabular}{|c|c|c|c|c|c|c|}
\hline & \multicolumn{3}{|l|}{ Urban } & \multicolumn{3}{|l|}{ Rural } \\
\hline & B & SE & p Value & $\mathbf{B}$ & SE & p Value \\
\hline \multicolumn{7}{|l|}{ Perceived health status } \\
\hline Good & Ref & - & - & Ref & - & - \\
\hline Normal & 0.681 & 0.096 & $<0.0001$ & 0.461 & 0.167 & 0.0057 \\
\hline Poor & 2.272 & 0.098 & $<0.0001$ & 1.826 & 0.165 & $<0.0001$ \\
\hline \multicolumn{7}{|l|}{ Level of social activity } \\
\hline None & Ref & - & - & Ref & - & - \\
\hline Moderate & -0.073 & 0.083 & 0.3803 & -0.724 & 0.285 & 0.0112 \\
\hline Active & -0.309 & 0.127 & 0.0148 & -0.102 & 0.153 & 0.5047 \\
\hline \multicolumn{7}{|c|}{ Perceived health status $\times$ social activity } \\
\hline Good×none & Ref & - & - & Ref & - & - \\
\hline Normal×moderate & -0.258 & 0.105 & 0.0141 & 0.036 & 0.184 & 0.8438 \\
\hline Normalxactive & -0.395 & 0.177 & 0.0256 & 0.205 & 0.393 & 0.6027 \\
\hline Poor $\times$ moderate & -0.629 & 0.107 & $<0.0001$ & -0.438 & 0.184 & 0.0171 \\
\hline Poorxactive & -1.087 & 0.264 & $<0.0001$ & -0.273 & 0.467 & 0.5587 \\
\hline \multicolumn{7}{|l|}{ Sex } \\
\hline Male & Ref & - & - & Ref & - & - \\
\hline Female & 0.201 & 0.075 & 0.0076 & 0.048 & 0.130 & 0.7104 \\
\hline \multicolumn{7}{|l|}{ Age (years) } \\
\hline $45-54$ & Ref & - & - & Ref & - & - \\
\hline $55-64$ & -0.148 & 0.055 & 0.007 & 0.062 & 0.111 & 0.5778 \\
\hline $65-74$ & 0.034 & 0.070 & 0.6303 & 0.189 & 0.128 & 0.1391 \\
\hline $75-84$ & 0.298 & 0.089 & 0.0009 & 0.296 & 0.151 & 0.0505 \\
\hline$>85$ & 0.369 & 0.152 & 0.0154 & 0.118 & 0.240 & 0.6240 \\
\hline \multicolumn{7}{|l|}{ Education } \\
\hline College graduate & Ref & - & - & Ref & - & - \\
\hline High school graduate & 0.097 & 0.086 & 0.264 & 0.168 & 0.235 & 0.4747 \\
\hline$<$ High school & 0.346 & 0.089 & $<0.0001$ & 0.403 & 0.223 & 0.0707 \\
\hline \multicolumn{7}{|l|}{ Income } \\
\hline Q4 (high) & Ref & - & - & Ref & - & - \\
\hline Q3 & 0.035 & 0.053 & 0.5035 & -0.093 & 0.114 & 0.4145 \\
\hline Q2 & 0.265 & 0.057 & $<0.0001$ & 0.008 & 0.112 & 0.9419 \\
\hline Q1 (low) & 0.259 & 0.064 & $<0.0001$ & 0.369 & 0.124 & 0.0029 \\
\hline \multicolumn{7}{|l|}{ Marital status } \\
\hline Married & Ref & - & - & Ref & - & - \\
\hline Divorced, widowed, unmarried & 0.588 & 0.077 & $<0.0001$ & 0.371 & 0.139 & 0.0077 \\
\hline \multicolumn{7}{|l|}{ Type of household } \\
\hline With family & Ref & - & - & Ref & - & - \\
\hline Alone & 0.178 & 0.097 & 0.0665 & 0.246 & 0.160 & 0.1234 \\
\hline \multicolumn{7}{|l|}{ Chronic disease } \\
\hline No & Ref & - & - & Ref & - & - \\
\hline Yes & 0.256 & 0.049 & $<0.0001$ & -0.015 & 0.083 & 0.8599 \\
\hline \multicolumn{7}{|l|}{ Employment status } \\
\hline Yes & -0.242 & 0.049 & $<0.0001$ & -0.568 & 0.076 & $<0.0001$ \\
\hline No & Ref & - & - & Ref & - & - \\
\hline \multicolumn{7}{|l|}{ Smoking status } \\
\hline Current smoker & Ref & - & - & Ref & - & - \\
\hline Ex-smoker & -0.137 & 0.078 & 0.0785 & -0.169 & 0.132 & 0.2014 \\
\hline Non-smoker & -0.174 & 0.076 & 0.0223 & -0.096 & 0.135 & 0.4740 \\
\hline \multicolumn{7}{|l|}{ Alcohol use } \\
\hline Current & -0.038 & 0.061 & 0.5376 & -0.116 & 0.105 & 0.2680 \\
\hline Past & 0.268 & 0.081 & 0.001 & 0.232 & 0.142 & 0.1039 \\
\hline Never & Ref & - & - & Ref & - & - \\
\hline
\end{tabular}


Table 5 Combined table of association between perceived health, social activity and CES-D10

\begin{tabular}{|c|c|c|c|}
\hline & $\mathbf{B}$ & SE & p Value \\
\hline \multicolumn{4}{|c|}{ Perceived health statusxsocial activity } \\
\hline Good×none & Ref & - & - \\
\hline Good×moderate & -0.071 & 0.073 & 0.3298 \\
\hline Goodxactive & -0.377 & 0.115 & 0.0011 \\
\hline Normalxnone & 0.626 & 0.083 & $<0.0001$ \\
\hline Normal×moderate & 0.372 & 0.073 & $<0.0001$ \\
\hline Normalxactive & -0.032 & 0.128 & 0.8043 \\
\hline Poorxnone & 2.140 & 0.084 & $<0.0001$ \\
\hline Poorxmoderate & 1.495 & 0.079 & $<0.0001$ \\
\hline Poorxactive & 0.868 & 0.206 & $<0.0001$ \\
\hline
\end{tabular}

All covariates in table 3 are adjusted.

CES-D10, Center for Epidemiological Studies Depression 10 Scale.

score than those who are not socially active. Therefore, the effect of social activity on the relationship between perceived health and CES-D10 score is increased in participants with poor perceived health.

In a subgroup analysis, we investigated the synergistic effect of social activity on the relationship between perceived health and CES-D10 by residential region: urban or rural. The synergistic effect of social activity appeared to be stronger in urban areas. As can be seen in table 4, the interaction term between perceived health and social activity was statistically significant for all perceived health/social activity combinations in urban residents, and addition of the interaction term decreased CES-D10 scores more than in the overall sample (table 3). By contrast, in rural residents, the interaction term between perceived health and social activity was only statistically significant for one perceived health/social activity combination. In addition, there were no consistent trends observed with regard to perceived health status, and most categories were not statistically significant. We assume that this may be due to different levels of intimacy in urban and rural settings. Beggs $e t a t^{22}$ suggested that personal networks in rural settings have greater intensity and role multiplexity. People in rural areas have a more intensive personal network, which may explain why they are less affected by social activity than urban participants. However, further study is required on the different effects of different kinds of social activity in urban and rural settings.

Previous studies have found inverse associations between social activity and depression and positive associations between perceived health and depression. In this study, we found that the interaction between these two factors had a synergistic effect on depressive symptoms. For example, in participants who reported poor health, the association between social activity and depressive symptoms was stronger than in those who reported good health. This synergistic effect appears to be stronger in urban areas. Thus, the effect of the interaction between social activity and perceived health should be considered when establishing health policies. Self-rated perceived health is known to be a prognostic indicator of depression. Our study results suggest that focusing recommendations for social activity on the people who have poor perceived health is effective. It can greatly improve their depressive symptoms and prevent their mental health from becoming worse; this intervention can therefore prevent progression to clinical depression.

This study has a number of strengths. To the best of our knowledge, it is the first to demonstrate the effect of an interaction between perceived health and social activity on depressive symptoms. Second, it analysed data

Figure 1 Adjusted least squares means for Center for Epidemiological Studies Depression 10 Scale (CES-D10) scores according to social activity level and perceived health status.

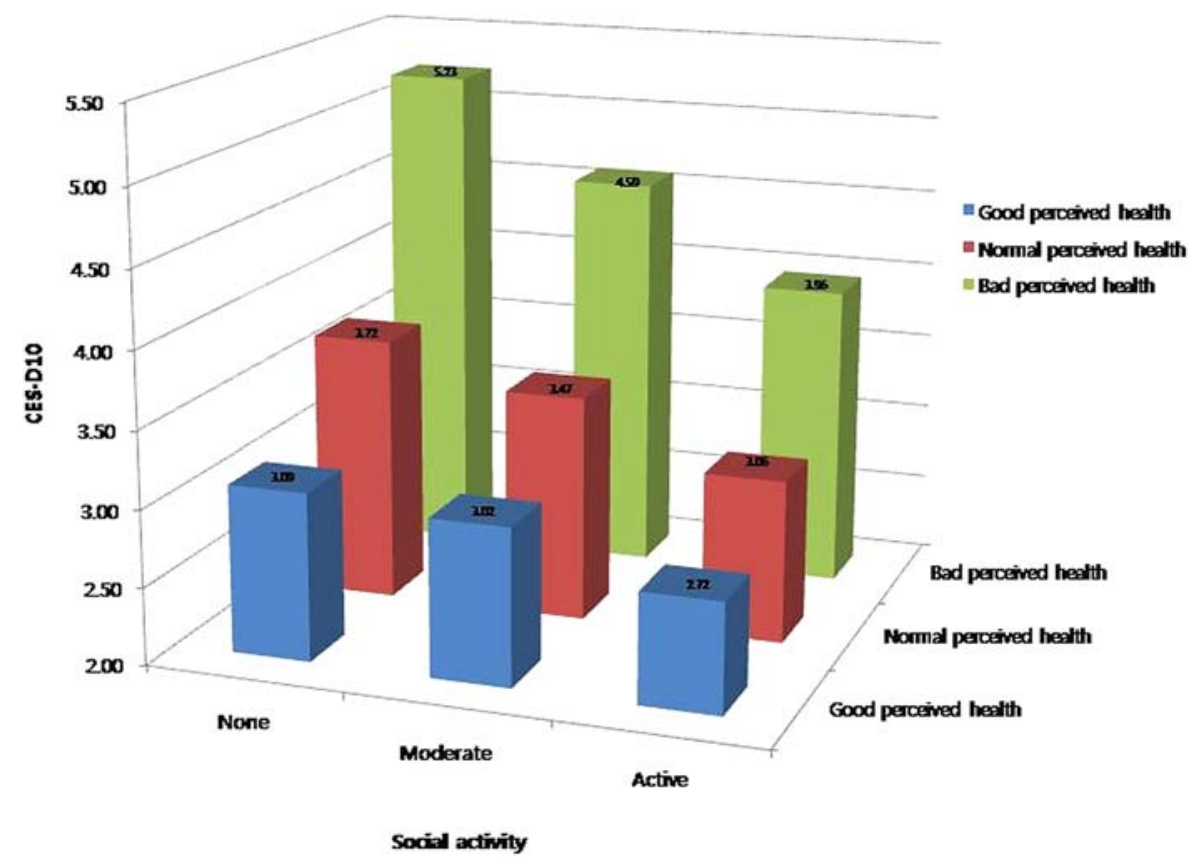


from a nationally representative sample. Finally, it had a large sample size. The study also has some limitations. First, social activity is just one way to measure social capital. Social networks, social support and social relationships were not considered in this study. Also, the frequency of social activity was not considered.

In conclusion, we found that the interaction between perceived health and social activity had a synergistic effect on depressive symptoms. Addition of this interaction term to our models resulted in a significant decrease in CES-D10 scores. Health policy makers should consider these associations when establishing health policies to address depression in later life among individuals with poor self-rated health.

\section{Author affiliations}

${ }^{1}$ Department of Public Health, Graduate School, Yonsei University, Seoul, Republic of Korea

${ }^{2}$ Institute of Health Services Research, Yonsei University, Seoul, Republic of Korea

${ }^{3}$ Department of Health Policy and Management, Graduate School of Public Health, Yonsei University, Seoul, Republic of Korea

${ }^{4}$ Department of Preventive Medicine, Yonsei University College of Medicine, Seoul, Republic of Korea

Contributors K-TH helped to design the study. SYL and COK helped to analyse and interpret the data. E-CP supervised and is the corresponding author. Our study met all the conditions that authorship credit requires.

Competing interests None.

Provenance and peer review Not commissioned; externally peer reviewed.

Data sharing statement Extra data can be accessed via the Dryad data repository at http://datadryad.org/ with the doi:10.5061/dryad.53cr4.

Open Access This is an Open Access article distributed in accordance with the Creative Commons Attribution Non Commercial (CC BY-NC 4.0) license, which permits others to distribute, remix, adapt, build upon this work noncommercially, and license their derivative works on different terms, provided the original work is properly cited and the use is non-commercial. See: http:// creativecommons.org/licenses/by-nc/4.0/

\section{REFERENCES}

1. OECD. Health at a glance 2013: OECD indicators. OECD Publishing, 2013.

2. NIH consensus conference. Diagnosis and treatment of depression in late life. JAMA 1992;268:1018-24.

3. Copeland JR. Prevalence of depressive illness in the elderly community. J R Coll Gen Pract Occas Pap 1987;(36):5-7; discussion 7-8.
4. Ambresin G, Chondros P, Dowrick C, et al. Self-rated health and long-term prognosis of depression. Ann Fam Med 2014;12:57-65.

5. Steger MF, Kashdan TB. Depression and everyday social activity, belonging, and well-being. J Couns Psychol 2009;56:289-300.

6. Isaac V, Stewart R, Artero S, et al. Social activity and improvement in depressive symptoms in older people: a prospective community cohort study. Am J Geriatr Psychiatry 2009;17:688-96.

7. Prince MJ, Harwood RH, Thomas A, et al. A prospective population-based cohort study of the effects of disablement and social milieu on the onset and maintenance of late-life depression. The Gospel Oak Project VII. Psychol Med 1998;28:337-50.

8. Adler G, Kriz D, Tremmel S. [The social situation of patients with late-life depression]. Psychiatr Prax 2003;30:207-11.

9. Kim JM, Shin I-S, Yoon J-S, et al. Prevalence and correlates of late-life depression compared between urban and rural populations in Korea. Int J Geriatr Psychiatry 2002;17:409-15.

10. Kohout FJ, Berkman LF, Evans DA, et al. Two shorter forms of the CES-D depression symptoms index. J Aging Health 1993;5:179-93.

11. Irwin M, Artin $\mathrm{KH}$, Oxman MN. Screening for depression in the older adult: criterion validity of the 10-item Center for Epidemiological Studies Depression Scale (CES-D). Arch Intern Med 1999;159:1701.

12. Tsai CF, Ouyang W-C, Chen L-K, et al. Depression is the strongest independent risk factor for poor social engagement among Chinese elderly veteran assisted-living residents. J Chin Med Assoc 2009;72:478-83.

13. Joiner TE Jr, Lewinsohn PM, Seeley JR. The core of loneliness: lack of pleasurable engagement-more so than painful disconnectionpredicts social impairment, depression onset, and recovery from depressive disorders among adolescents. J Pers Assess 2002;79:472-91.

14. Rosso AL, Gallagher RM, Luborsky M, et al Depression and self-rated health are proximal predictors of episodes of sustained change in pain in independently living, community dwelling elders. Pain Med 2008;9:1035-49.

15. Lyness JM, King DA, Conwell Y, et al. Self-rated health, depression, and one-year health outcomes in older primary care patients. $A m J$ Geriatr Psychiatry 2004;12:110-13.

16. Zuccala G, Cocchi A, Carbonin P. The impact of depression on self-perceived health status. J Am Geriatr Soc 1995;43:198-9.

17. Choi KS, Stewart R, Dewey M. Participation in productive activities and depression among older Europeans: survey of health, ageing and retirement in Europe (SHARE). Int $J$ Geriatr Psychiatry 2013;28:1157-65.

18. Ahern MM, Hendryx M. Community participation and the emergence of late-life depressive symptoms: differences between women and men. J Womens Health (Larchmt) 2008;17:1463-70.

19. Arai A, Ishida K, Tomimori M, et al. Association between lifestyle activity and depressed mood among home-dwelling older people: A community-based study in Japan. Aging Ment Health 2007;11:547-55.

20. Hahn CY, Yang M-S, Yang M-J, et al. Religious attendance and depressive symptoms among community dwelling elderly in Taiwan. Int J Geriatr Psychiatry 2004;19:1148-54.

21. Mechakra-Tahiri SD, Zunzunegui MV, Préville M, et al. Gender, social relationships and depressive disorders in adults aged 65 and over in Quebec. Chronic Dis Can 2010;30:56-65.

22. Beggs JJ, Haines VA, Hurlbert JS. Revisiting the rural-urban contrast: personal networks in nonmetropolitan and metropolitan settings. Rural Sociol 1996;61:306-25. 